\title{
A Review on Health-Care Acquired Infections of the Hospital Staff during Routine Patient's Delivery Service
}

\author{
${ }^{1}$ ENAIGBE, AA; ${ }^{2}$ IRODI, CC
}

\author{
${ }^{*}$ Department of Biological Sciences, College of Natural and Applied Sciences, Igbinedion University, Okada, Nigeria \\ ${ }^{2}$ Department of Nursing, College of Basic Health Sciences, Igbinedion University, Okada, Nigeria \\ *Corresponding Author Email: andrewenaigbe89@ gmail.com; Tel: +2348131230105
}

\begin{abstract}
The health-care acquired infections (HCAIs) occur world-wide among persons undergoing medical attention in health institutions and result in unexpected long-term stay, disability and financial loses. The most predominant infections are catheter associated urinary tract, central line associated, surgical site and ventilator associated pneumonia infections. The patients are prone to infections during hospitalization from varied environmental sources, hands of health-care professionals, medical equipment and other infected patients. The frequent factors affecting patients on admission are improper hand hygiene, contact with infected patients, adverse drug events and surgical complications. Patients under health-care delivery can acquire infection disseminated from food, water, aerosols and hospital wastes. The application of personal protective equipment, routine educational interventions are common approaches that can help stop HCAIs and save lives, decrease death rate and health delivery expenses. In buttressing this, the World Health Organization (WHO) enunciated guidelines to enhance hand washing practices, infection prevention and control programme, monitored use of antibiotics and its resistance. The other measures included global adoption of efficient surveillance system and the impact of relevant stakeholders in health sectors needed to prevent and control hospital acquired infections.
\end{abstract}

\section{DOI:https://dx.doi.org/10.4314/jasem.v25i9.6}

Copyright: Copyright (C) 2021 Enaigbe and Irodi. This is an open access article distributed under the Creative Commons Attribution License (CCL), which permits unrestricted use, distribution, and reproduction in any medium, provided the original work is properly cited.

Dates: Received: 09 May 2021; Revised: 12 August 2021; Accepted: 12 September 2021

Keywords: Control strategies, environment, Infection, health-care, hospital staff and pathogens

The health-care acquired infections (HCAIs) "nosocomial infections" occur in individuals undergoing medical attention in an hospital or similar health institutions which was not present during hospitalization. It can exist during service delivery and even after the discharge of the patients. The term HCAIs originally described as infections linked with patients on admission to health-care facility. Microbial contamination can be acquired from an inanimateobjects that had been previously polluted from a human source (Environmental infection), carried by the patient prior to hospitalization (Self infection) and obtained from someone in the hospital (Cross infection). These modes of infections can be constituted to a procedure conducted in the hospital. However, most of these diseases acquired in the health facilities are extremely different and classified into: septic disease, with principal properties of local acute inflammation, wound infection, respiratory and urinary tract infections; diarrhea characterized by intestinal tract infections and the ordinary infectious fevers, manifesting as influenza or measles. The organization and activities of the hospital determine the pattern and distribution of HCAIs. The predisposing threatening conditions to nosocomial infections are based on the environment that concerns poor hygienic factors and inadequate waste disposal from health-care facilities, compromised immunity in patients, prolonged stay in intensive care unit (ICU), and improper application of invasive devices, injection techniques and unawareness of primary control strategies. The US Center for Disease Control noted that, approximately 2 million hospitalized patients get HCAIs annually, while being treated for other health challenges and more than 120,000 have died due to HCAIs. More so, the HCAIs comprise of hazards in medical health facilities through invasive devices such as catheters, ventilators and surgical equipment (CDC. 2016). The group of persons at risk are patients in intensive care units (ICUs), burns, neonates, undergoing organ transplant, lack of awareness and finances. From time in-memorial, there has been the reasoning that the embrace of medicine can be detrimental and contrarily be beneficial to mankind. In two centuries ago; Hipppocrates, the originator of modern medicine stated that "I will apply medication to heal the sick in accordance with my capacity as a physician and deprive them of that which can be harmful" The idea that medicine could be detrimental was considered an un-expectant physical harm arising from or attributed to by health -care service Owens, 2008).Therefore, nosocomial infections being a significant source of illness and death should be eliminated from the base-line so that their dissemination can be mitigated. The HCAIs are a great safety risk for both health service deliverers and patients due to its outrageous rate leading to increased morbidity, mortality, prolonged hospitalization, and 
health service costs (Hassan et al., 2016). Therefore, numerous Health bodies advocated that, to avoid HCAIs which form colonies with multi-drugs resistant organisms, individuals should maintain the most single practice of effective hand hygiene (WHO, 2018).

Epidemiology and Prevalence: A physician by name, Phillip Semmelweis who has largely considered and acknowledged that, the health-care givers could contaminate disease, observed higher rates of death among pregnant women cured by obstetricians than those treated by midwives. He noted that, a pathologist wounded himself with a scalpel and died while performing an autopsy on a patient diagnosed of puerperal sepsis. Therefore, he revealed that, both a scalpel and the hands of obstetricians could transmit organisms causing infections to mothers in labour room or undergoing caesarean operation (Mitchell et al., 2016). More so, the other means of transmission are through faecal-oral, hand to mouth and inhalation of respiratory aerosols of infected patients when coughing, sneezing and talking. Several incidences of infectious diseases in hospitals are not epidemiologically different from outbreaks in other comparable institutions such as hotels, nurseries or schools. The patients in hospital environment using same supply of water and food, and where members of this community are in proximal contact to each other often result in outbreaks. In a survey analysed in 162 US hospitals with 10,115 patients reported that, at minimum, $5 \%$ of screened patients recorded one HCAI, with the most distributed organism being Staphylococcus aureus. The commonest infections were gastrointestinal infections, surgical site infections (SSIs) and pneumonia. After 2 years, the same group discovered that, $5 \%$ (57) of patients had suffered from HCAIs with the upper most $68 \%$ acquiring blood stream infections and urinary tract infections (UTIs) with the most prevalent organism being Clostridium difficile. In another report, it was revealed that, the total mean microbial value was 8.56 $\pm \log _{10} 5 \mathrm{cfu} / \mathrm{ml}$. The highest value (32) pathogens from door handles and the least number of pathogens recorded on hospital floors (07). The isolated bacteria were Bacillus subtilis, Pseudomonas aeruginosa and Staphylococcus aureus.

Factors that can influence risk of infection: Microbes that are usually found in the ecosystem are the causal agents of hospital acquired infections. The basic fundamental parameter determining the occurrence of pathogenic infection is the seldom interaction persons with the microorganisms. Five basic associated conditions that facilitate the distribution and nature of infections include:

Contact with infectious individuals: The infectious persons are both admitted and discharged by health facilities because, potential sources of infection for others are the patients with infectious ailments and carriers of virulent organisms that are received in health-care homes or isolation centres. An additional source or new infection is produced by patients who have become contaminated in hospital. As a result of routine procedure and infrastructural organization of hospital setting, the patients with similar elevated susceptibility level to disease tend to be situated in a particular section; the burned patients, those with urological infections, new born infants and human immuno-deficiency virus (HIV) disease. The standardization and frequent repetitive nursing procedures tend to promote various favourable conditions from the transmission of organisms by direct interaction from an infected person to another (Hassan et al., 2016).

Infected environmental sites: Microorganisms which tend to be transmitted to receptive host sites on persons occur through certain contaminated materials and objects. The evidence of microbial aetiology suggests that several diseases originating from air, dust and surfaces are due to organisms that contaminated them slightly recently. These infected sites are considered temporary reservoirs and the infection arising, is termed cross infection. The extraneous environmental sources from patient body flora, post-operative tetanus operation derived from contaminated substances and improperly sterilized process prior to hospitalization are a major way for polluted site (Medina-Ramon, 2005). Microorganisms capable of growing in moist and fluid conditions often survive for long duration, can proliferate at these sites in the presence of restricted nutrient composition. Poor sanitary practices and indiscriminate waste disposal is also regarded a risk factor.

Unawareness: In low income Nations of the world, lack of funds, poverty, illiteracy, insufficient health personnel and unavailability of hospital equipment are risk-linked factors. The other threat-prone conditions are; inappropriate application of invasive devices, improper use of injection, lack of control policies and poor knowledge of basic infection control (WHO, 2018).

Reduced intolerance to infection: The pre-existing infection for which persons are admitted, medical and surgical therapeutics administered and age are responsible for reduced resistance to infection by many hospitalized patients. The underlying infection, drug treatment, irradiation and new-born infants are endowed with lower resistance to disease. In this situation, the pervading microbes from the body surface can easily attack, penetrate, and destroy the tissues, thereby leading to disease formation due to their weakened immune system (Avelar-Pires et al., 2004).

Microbial drug resistance: Antimicrobial therapeutics are administered to a large number of hospitalized patients to suppress the body micro flora that are 
sensitive to the drug and in the process, the resistant strains become endemically established in the hospital population, due to mutational changes in microbial cell wall. This enables the treatment not to be accomplished and reduce the drug potency (AvelarPires et al., 2004; WHO, 2014). The patient can become a source of infection, if he becomes a carrier of a resistant pathogen and subsequently, uncontrolled occurrence and growth of the organism may enhance patient infectivity, if he receives the antibiotic to which the organism is not susceptible (Justo and Bokstaven, 2014).

HCAIs associated microorganisms: The microorganisms responsible for HCAIs are classified broadly into the following groups: Conventional, where organisms cause infection in healthy individual; Conditional, pathogens cause disease only in patients with lowly resistance to infection and the opportunistic group, these are microbes, whose activities result in disease, but only in patients with highly decreased resistance with generalized suppressed immunity (Akbari and Kyellerup, 2015).

The primary groups of microbial causes of HCAIs include: Bacteria: The most prevalent organisms causing hospital-care associated infections are the bacteria and mostly occur in the normal flora of an individual with weakened immune system. The group of bacteria mainly found in intensive care unit causing infection is the Acinetobacter. It has been implicated for $76.0 \%$ of recorded cases occurring in dust and water. The methicillin-resistant Staphylococcus aureus (MRSA) is distributed through direct contact, open wounds and contaminated hands. It is responsible for leukaemia, pneumonia and sepsis and highly resistant to the $\beta$-Lactams antibiotics (CDC, 2016; Hassan et al., 2016). In general, other bacteria usually present in hands of staff and hospital environment include; Streptococci A and B groups, Salmonella spp., Flavobacterium meningosepticum, Corynebacterium diphtheria, Mycobacterium tuberculosis and Bordetella pertussis.

Fungi: Previous studies had identified some fungi frequently isolated in health-care settings to include; Aspergillus flavus, Candida albicans and Cryptococcus sp. The concomitant infections of Aspergillus are caused by breathing in of fungal spores from polluted air and has been dduced to the death of nosocomial infectious patients. Aspergillus flavus has been implicated in Covid-19 incidences and related diseases such as pulmonary disorders and allergies. The pathobiont (Candida albicans) found in many healthy persons from patient's endogenous microflora and orally transmitted can become invasive to life debilitating systemic infections. More so, Cryptococcus sp. (C. neoformans) C. meningitis that spreads from the lungs to the brain after inhaling dust contaminated by faeces of birds. The disease is characterized by chest pain, cough, pneumonia and seizure that are principal symptoms of Corona virus disease (Akbari and Kjellerup, 2015; CDC, 2020). However, other fungal groups found to be linked with HAIs were; Nocardia, Moulds, Histoplasma and Toxoplasma.

Table 1. The main microorganisms responsible for HCAIs

\begin{tabular}{lll}
\hline $\begin{array}{l}\text { Class of } \\
\text { microorga } \\
\text { nism }\end{array}$ & Type of organism & Pathogenicity \\
\hline Bacteria & Staphylococcus aureus & \\
& Streptococcus type A and B & $\mathrm{P}, \mathrm{O}$ \\
& Enterococci & $\mathrm{C}$ \\
& Clostridium tetani & $\mathrm{C}$ \\
& Escherichia coli & $\mathrm{P}$ \\
Fungi & Pseudomonas aeruginosa & $\mathrm{C}$, \\
& Mycobacterium tuberculosis & $\mathrm{P}$ \\
& Bordetella pertussis & $\mathrm{P}$ \\
& Corynebacterium diphtherae & $\mathrm{P}, \mathrm{C}$ \\
& Mycobacterium tuberculosis & $\mathrm{P}$ \\
& Candida albicans & $\mathrm{C}, \mathrm{O}$ \\
& Cryptococcus & $\mathrm{P}, \mathrm{O}$ \\
Viruses & Nocardia, moulds & $\mathrm{O}$ \\
& Hepatitis, Smallpox & $\mathrm{P}$ \\
& Herpes simplex,Toxoplasma & $\mathrm{P} \mathrm{C} \mathrm{O}$ \\
& Cytomegalovirus & $\mathrm{P} \mathrm{C} \mathrm{O}$ \\
& Chickenpox, Influenza & $\mathrm{P}$ \\
& Rubella & $\mathrm{P}$ \\
Rotaviruses & $\mathrm{P}$ \\
\hline
\end{tabular}

Source: (CDC, 2016; Hassan et al., 2016): Key: $P=$ Conventional pathogen; causes clinical disease in healthy persons. $C=$ Conditional pathogen; causes diseases only in presence of specific predisposing factor. $O=$ Opportunist pathogen results in generalized disease only in patients profoundly reduced resistance to infection.

Viruses: The opportunistic infections due to viruses are disseminated from one person to another through faecal-oral, hand-mouth and respiratory accesses (Lai et al., 2020). The commonest chronic viral arising from unsafe injection practices are, Hepatitis B and C. The viruses are able to kick-start infection, spread 
throughout body and multiply as a result of particular virulence factors such as surface proteins and co receptors. The other viruses include Cytomegalovirus, Chicken pox, Corona virus, Herpes simplex, Human immune deficiency (HIV) Rota virus and small pox (Kampf et al., 2020).

Transmission: The unhygienic conditions of the health-care immediate environment during health-care service delivery; the staff hands, floor, air, food and water can be polluted and distributed thereafter (Kampf et al., 2020).

Environment: During hospitalization, the spread to other patients enables one to become a better source for an uninfected individual. The health-care environment constituting the equipment, beds, baths, toilet floor, food, water and other medical devices can be a pathway for transmission of pathogens living in the health-care surrounding.

Hospital waste management: The deposited waste originating from health-care institution is regarded as hazardous and contained waste components of heavy metals, contaminated blood and sputum and improper handling of hospital waste can be considered as a habitual storage-place for pathogens.

Patients and staff contact: The direct personal contacts of staff with the patient's respiratory aerosols, hands, saliva and other body fluids, unsterilized equipment, improper hand washing and imperfect use of PPE are capable of promoting the dissemination of pathogens during hospitalization.

Patient's microflora: Infections can occur if the normal endogenous microflora of persons are transmitted to tissue wounds, surgical sites and other body parts. The microorganisms found in the human digestive tract such as the Clostridium listeria, Escherichia coli, Klebsiella biotypes and Streptococcus A and B types can cause surgical site infection (SSI), wet and dry hospital environments can act for microbial growth and survival.

Types of Health-care acquired infections (HCAIs): Cross contamination between patients and health providers, improper and prolonged use of implants and prostheses usually play significant impact in HCAIs and the various kinds include: (Christine and Elizabeth, 2017).

Central line-associated blood stream infections (CAUTIs): The central line-associated blood stream infections (CLABSIs) has a high morbidity incidence rate and regarded as a deadly type of opportunistic hospital infections. In this case, catheters are inserted in central lines to administer drugs and fluids while continuous application can result in severe contaminations of the blood stream leading to weakened health body system and high care cost. The most frequent pathogenic agents are the Staphylococcus aureus, Enterococcus and Candida albicans. (Michell et al., 2016). Several risk factors are concerned with high cases of CLABSIs and these include; increased length of stay before and after insertion of central venous catheters (CVCs), multiple CVCs, and parenteral nutrition, increased CLABSIs rates in children and neonates and presence of underlying diseases such as hematologic, cardiovascular and gastrointestinal infections. Various types of CVCs that are used in hospitals are indicated in table 2.

Table 2: Types of central venous catheters (CVC).

\begin{tabular}{|l|l|l|l|}
\hline Catheter & Period of use & Insertion & CLABSI Risk \\
(i). Non-tunnelled & Short-term & Percutaneous & Accounts for most CLABSI \\
(ii). Tunnelled CVC & Long-term & Surgical & Lower rate \\
(iii) Implantable ports & Long term & Surgical insertion and removal & Lowest risk \\
(iv). Peripheral CVC & Short to intermediate & Bedside insertion & Lower risk \\
\hline
\end{tabular}

(Source: Michell et al., 2016). Key: CLABSI, Central line associated bloodstream infection; CVC, Central venous catheter.

Catheter-associated urinary tract infections (CAUTIs): Indwelling urinary catheters are drainage tubes that insert into the urethra, positioned in the urinary bladder and are linked to an enclosed receiving system to drain urine. This infection is caused by indigenous microflora of the patient's meatus, rectum, urethra and vagina. It accounts for about $36 \%$ of recorded HCAIs with huge impacts for death rate and financial costs and commonest type of nosocomial infection worldwide according to acute care health statistics (Cassini et al., 2016). Inserted catheters act as an access for bacteria penetration while some quantity of urine retained in the bladder arising from breached drainage, harbour and enhance the bacteria to strive. The risk factors concerned with CAUTIs included older age, prolonged catheter stay, diabetics mellitus and absence of systemic antibiotic administration, that often lead to advanced bacterial resistance and resulting in biofilm formation, which tends to defend microbes from both antimicrobials and host defence mechanisms. It has also been implicated with cystitis, epididymitis, prostatitis (in males) and meningitis. The microbes usually connected with CAUTIs are Escherichia coli identified as the most infecting pathogen, Enterococcus, Pseudomonas aeruginosa, Klebsiella pneumonia and Enterobacter (CDC., 2016). The symptoms include; fever $>38^{\circ} \mathrm{C}$, 
urinary urgency, frequency and dysuria (Akbari and Kyellerup, 2015; WHO, 2018).

Surgical site infections (SSIs): The endogenous microflora is responsible for most surgical site infections, second frequent type of opportunistic infection primarily caused by Staphylococcus aureus resulting in long hospital stay and death. Individuals at risk are those with underlying conditions that include; diabetics, intraoperative blood transfusion and steroid intake. More so, the SSI patients have the highest risk for cardiac, intra-abdominal surgery and orthopaedic and about $20 \%$ of Persons with SSIs develop acute sepsis and shock. At the end of incision, all surgical injuries have some level of infection and some factors such as surgical room environment, the host, specific pathogen and operating process facilitate SSIs pathogenesis (CDC, 2016). A study indicated a case of
SSIs in orthopaedic patients with the commonest organism being Staphylococcus species including MRSA, Acinetobacter species, Pseudomonas species and Enterococcus species (CDC, 2016).

Ventilator associated pneumonia (VAP): It occurs in individual placed on assisted automated ventilator device to enhance proper intake of oxygen in patients experiencing difficulty in breathing. The symptoms include; altered bronchial vibrations, fever and leucopenia and changes in sputum. Several studies have identified the Gram-negative bacilli as most predominant organisms, followed by Pseudomonas and Klebsiella species. Other organisms associated are vancomycin-resistant Enterococcus, Staphylococcus aureus, Acinetobacter baumannii and Serratia marcescens (Lai et al., 2020).

Table 3: Health-care associated infections, incidence and prevalence

\begin{tabular}{|l|l|l|l|}
\hline HCAI & Mortality rate (\%) & 2019 SIR & 2019 VS 2018 SIR (\%) \\
CLABSI & 20.2 & 0.70 & 10.0 \\
CAUTI & 4.3 & 3.0 & 7.0 \\
SSI & 5.2 & 2.7 & 7.0 \\
\cline { 1 - 1 } VAP & 13.0 & NR & NR \\
\hline \multicolumn{2}{|l|}{$(C D C, 2016)$. Key: SIR, standardized infection ratio; NR, not reported }
\end{tabular}

Prevention and control strategies of HCAIs: The transmission of HCAIs has to be curbed from the primary source for being responsible for most illnesses and deaths in the society. The HCAIs exhibit an interaction with the multidrug resistance (MDR) organisms, therefore, the WHO has advocated that, frequent and proper hand washing concept, is the single effective practice to mitigate its debilitating impact on humans (Hassan et al., 2016). Subsequently, applying cleaning agent (antiseptics) on baths, beds, floor, toilets, surfaces and walls and several other medical devices should be enshrined in health-care policy guidelines. Air-borne bacterial infections can be eliminated by constant monitoring of air filters and ventilation system of the general wards, surgical theatre rooms and intensive care units (ICU). The microbiological analytical methods should be applied for the food and water analyses, while their clean and quality production systems and environmental conditions are maintained and adherence to basic standard parameters (Rutala and Weber, 2013; Cassini et al., 2016). Similarly, the proper use of personal protection equipment (PPE) such as face masks, hand gloves, gowns, head covers and protective eye wears. In addition, other approaches to combat HCAIs include; proper injection-giving practices, working equipment sterilization should be emphasized and made compulsory to health-care service deliverers, transmission-based precaution, immunization and training of health-care workers. Restricted areas should be earmarked for storage or deposition of perceived infectious health-care waste products and hospital workers should also be aware of the dangers associated with waste materials. The health-care authorities should embark on effective management approaches in prevention of contaminations and an infection control programme. Consequently, the application of drugs, and vaccines are used in the protection and control of respiratory diseases, whose pathogenic agents are Bordetella pertussis, influenza virus, Haemophilus influenzae, severe acute respiratory syndrome-associated corona virus (SARSCOV) and Mycobacterium tuberculosis. These pathogens are easily released while coughing, sneezing and talking as aerosols or droplets in closed health-care facilities and in over-crowded settings. To efficiently terminate virulent hospital microbes sticking to surfaces in hospital room and equipment, ultraviolet devices and hydrogen peroxide vapour technologies antimicrobial therapeutics can be successfully applied to sterilize areas presumed to be colonized by the pathogenic organisms. Inadequate application of antimicrobial has led to several death globally due to emerging and re-emerging drug resistant microorganisms caused by antibiotics selfprescription, mis-use, dosage, over-prolonged application and inadequate standard guidelines for health professionals. Several cases of methicillin resistance Staphylococcus aureus (MRSA) and other multi-drug resistant Gram-negative bacteria are largely responsible for increased rate of HCAIs (Justo and Bokstaven, 2014). Subsequently, action plan is required not to negate the goals so far achieved in the implementation of IPC program as enunciated by the WHO, which included better hygiene practices, availability of clean water and vaccination to reduce the need of antibiotic dependence. More so, to be aloft of emerging antibiotic deleterious health consequences, there should be formation of recent diagnostics advances and collaboration among policy 
makers to foster cooperation and data dissemination. Sequel to the foregoing, the epidemiological surveillance for the determination of level of achievement of IPC program is desirable and the proper survey techniques included: data collection which comprises of; administrative information, risk factors, patient's record, laboratory test and data validation. This is aptly followed by feedback and results that involved, information dissemination, control, confidential keeping of patient's laboratory records and intermittent collection of data for efficient surveillance analysis (CDC, 2016; Hassan et al., 2016).

Conclusion: The HCAIs stand as the leading cause of mortality and morbidity due to the increase of antibiotic-resistant bacteria and reluctance by healthcare providers to strictly adhere to the principles of infection protection and control practice. The implementation of best health practice involves corresponding technique for therapeutic use, appropriate epidemiological surveillance system and biosafety program, adequate waste disposal methods, policy reforms and general awareness can assist in curbing the debilitating impacts of HCAIs.

\section{REFERENCES}

Akbari, F and Kyellerup, B (2015). Elimination of bloodstream infections associated with Candida albicans biofilms in intravascular catheters. Pathogens 4(3): 457 - 469

Avelar-Pires, CA; Ferreira, SN; Montero, L (2004). Clinical epidemiological and therapeutic profile of dermatophyte. Annual Brazillian Dermatology. 89(2): $259-256$

Cassini, A; Hathaway, S; Havelaar, A; Koopmans, M; Koutsoumanis, K; Messens, W; Scheutz, F (2016). Microbiological risk assessment. European Food and Safety Association 4: 1 - 10

Center for Disease Control (CDC) (2016). Diseases and organisms in health-care settings: Health-care associated infections (HCAIs). Atlanta, Georgia.
[Online] Available from: https://www. Cdc.gov/hai/organisms/organisms.html

Christine, B and Elizabeth, K (2017). Hospital acquired infections: Current trend and infection. Journal of Critical Care Nursing 29: 51 - 65

Hassan, AK; Fatima, KB; Riffat, M (2016). Nosocomial infections: Epidemiology, prevention, control and surveillance. Asian Pacific Journal of tropical biomedicine 10: 1 - 5 .

Justo, JA; Bokstaven, PB (2014). Antibiotic lack therapy: a review of technique and logistical challenges. Infection and Drug Resistance 7: 342 $-346$

I

Kampf, G; Todt, PG; Pfaender; S; Steinmann, E (2020). Persistence of coronavirus on inanimate surfaces and their interaction with biocidal agents. Journal of Hospital Infections 104: 246 - 251

Lai, CC; Shih, TP; Tang, H.J; Hsueh, P.R (2020). Severe acute respiratory syndrome coronavirus 2 (SARS-Cov-2) and coronavirus disease. (http://doi.org/10.1016/j.ijantimicag.2020.10592 4).

36/oem.2004.017640.

Mitchell, B; Ferguson, J; Anderson, M (2016). Length of stay and mortality associated with health-care urinary tract infections: a multi-state model. Journal of Hospital Infections 92(1): 92 - 99.

Rutala, WA and Weber, D.J. (2013). Disinfectants used for environmental disinfection and new room decontamination technology. American Journal of Infection Control 41: $36-41$.

World Health Organization (WHO) (2018). Preventing bloodstream infections from centralline venous catheters. Geneva. [Online] Available from: https://who.int/patientsafety/implementation/bsi/ en/ 